\title{
Corrigendum
}

\section{Corrigendum to: Minimum requirements for the estimation of the measurement uncertainty: Recommendations of the joint Working group for uncertainty of measurement of the CSMBLM and CCMB}

\author{
Ivana Ćelap*1,2, Ines Vukasović1,2, Gordana Juričic ${ }^{13}$, Ana-Maria Šimundic ${ }^{1,4}$
}

${ }^{1}$ CSMBLM, Committee for the scientific professional development, Working group for uncertainty of measurement of the CSMBLM and CCMB, Croatia

${ }^{2}$ Clinical Institute of Chemistry, University Hospital Centre Sestre milosrdnice, Zagreb, Croatia

${ }^{3}$ Department of Laboratory Diagnostics, General Hospital Pula, Pula, Croatia

${ }^{4}$ Department of Medical Laboratory Diagnostics, Clinical Hospital Sveti Duh, Zagreb, Croatia

*Corresponding author: ivana.celap@gmail.com

This is a correction of Biochemia Medica 2017;27(3):030502. DOI: https://doi.org/10.11613/ BM.2017.030502.

Since the publication of the article "Minimum requirements for the estimation of the measurement uncertainty: Recommendations of the joint
Working group for uncertainty of measurement of the CSMBLM and CCMB", the authors have noticed that one of the equations presented in Appendix 1 (Example 2) for trueness calculation was published incorrectly. The correct equations for Appendix 1, Example 2 are presented below. The authors apologize for any inconvenience caused to the readers.

Appendix 1. Examples of measurement uncertainty estimation

EXAMPLE 2. Measurement uncertainty estimation including bias (if CRM is used)

\section{TRUENESS}

\begin{tabular}{cc}
\hline $\begin{array}{c}\text { Target value from manufacturer, } X_{\text {ref }} \\
\mathrm{B}=\bar{X}-X_{\text {ref }}\end{array}$ & 5.40 \\
$\left.\mathrm{~B}_{\text {rel }}=\left(\bar{X}-X_{\text {ref }}\right) / X_{\text {ref }}\right) \times 100$ & $1.7 \%$ \\
\hline 2. UNCERTAINTY OF MEASUREMENT & $5.08 \% \cong 6 \%$ \\
\hline$U_{\text {rel }}=2 \times \sqrt{\mathrm{B}_{\text {rel }}^{2}+\mathrm{U}_{\text {rel }}^{2}}$ & \\
\hline
\end{tabular}

The acceptance of the result is verified trough comparison with the TE value (6.96\%).

CRM - certified reference materials. 